\title{
The de novo Q1042R POGZ mutation in sporadic ASD disrupts the neuronal differentiation
}

\author{
Kensuke Matsumura ${ }^{1}$, Takanobu Nakazawa ${ }^{1,2}$, Shota Okada ${ }^{1}, K_{\text {Kazuki Nagayasu }}^{1,3}$, Hiroki Miura ${ }^{1}$, \\ Atsushi Kasai ${ }^{1}$, Kazuhiro Takuma ${ }^{2,4}$, Hidenaga Yamamori ${ }^{5}$, Yuka Yasuda ${ }^{5,6}$, Ryota Hashimoto ${ }^{4,5}$, \\ Hitoshi Hashimoto ${ }^{1,4,7,8}$
}

\author{
${ }^{\prime}$ Laboratory of Molecular Neuropharmacology, Graduate School of Pharmaceutical Sciences, Osaka University, Japan, \\ ${ }^{2}$ Department of Pharmacology, Graduate School of Dentistry, Osaka University, Japan, ${ }^{3}$ Department of Molecular \\ Pharmacology, Graduate School of Pharmaceutical Sciences, Kyoto University, Japan, ${ }^{4}$ Molecular Research Center for \\ Children's Mental Development, United Graduate School of Child Development, Osaka University, Japan, Department \\ of Psychiatry, Graduate School of Medicine, Osaka University, Japan, ${ }^{6}$ Oncology Center, Osaka University Hospital, \\ Japan, 'iPS Cell-Based Research Project on Brain Neuropharmacology and Toxicology, Graduate School of \\ Pharmaceutical Sciences, Osaka University, Japan, ${ }^{8}$ Division of Bioscience, Institute for Datability Science, Osaka \\ University, Japan
}

Autism spectrum disorder (ASD) is a neurodevelopmental disorder characterized by impairments in social interactions, reduced verbal communication abilities, stereotyped repetitive behaviors and restricted interests. Although genetic studies have identified numerous candidate genetic variants, the molecular etiology of ASD remains poorly understood. Recent studies have suggested that de novo mutations contribute to the risk of ASD. In particular, genes with highly recurrent de novo possible loss-of-function mutations, which are likely to play key roles in the etiology of ASD, have been identified in multiple unrelated patients. Among these high-confidence ASD risk genes, POGZ is one of the most recurrently mutated genes in ASD patients; we and other groups have recently identified at least 20 independent de novo possible loss-offunction mutations in POGZ. Therefore, de novo POGZ mutations can be related to ASD pathogenesis. However, the precise role of POGZ in the brain remains unknown. Here we present the role of POGZ in the neuronal differentiation and the functional characterization of de novo POGZ mutations.

We found that Pogz was highly expressed in the developing mouse cerebral cortex during embryonic neurogenesis and that POGZ knockdown impaired the neuronal differentiation. Since POGZ contains a centromere protein-B-like DNAbinding (CENPB-DB) domain and is likely to function as a chromatin regulator and a transcription factor, we examined the effect of the de novo mutations on the DNA-binding activity of POGZ as assessed by co-precipitation with DNA fragment carrying the CENP-B box sequence. We found that the de novo Q1042R mutation within the CENPB-DB domain of POGZ that we recently identified in a sporadic ASD case resulted in a reduction of approximately $60 \%$ in the DNA-binding activity of POGZ. To examine the functional significance of the Q1042R mutation in patient's neural stem cells, we established iPS cells from the patient who has the Q1042R mutation. We found that the patient's neural stem cells showed impaired neuronal differentiation.

Our results suggest that ASD-associated de novo mutations impair the DNA-binding activity of POGZ, an effect likely to result in the perturbation of chromatin function and the neuronal differentiation. These findings provide important insights into the cellular basis of ASD. 